\title{
A Survey on Rough Intervals Programming Formulation and Methodologies
}

\author{
Osama M Abou-Seida ${ }^{1}$ \\ Computer Science Department, \\ Faculty of Computers and Information Sciences, \\ Kafrelsheikh University, Egypt.
}

\author{
A. A. Abohany ${ }^{2}$ \\ Information Technology Department \\ Faculty of Computers and Information Sciences, \\ Kafrelsheikh University, Egypt. \\ E-mail: amrabohany8@gmail.com
}

\begin{abstract}
This research states a survey on formulation of linear programming problems with rough intervals coefficients in the objective functions and constraints, basic preliminaries about rough intervals, interval method and trust probability constraints for transforming rough intervals to crisp nature and fully rough intervals problems. Finally, presents different operational research models that contain rough intervals coefficients.
\end{abstract}

Keywords: rough intervals, interval method, trust probability constraints and fully rough intervals.

\section{Introduction}

The Rough Intervals (RIs), proposed by Robolledo [14] in 2006, are used to deal with partially unknown or illdefined parameters and variables. RI is introduced to adapt the rough set principles to model continuous variables. It is notable that rough sets were used only to handle discrete objects, initially, and could not represent continuous values. RI is a particular case of rough sets. It fulfils all the rough sets' properties and core concepts, including the upper and lower approximation definitions.

RI which based on the interval analysis has been created as a helpful and basic method to deal with the classificatory analysis of ambiguous concepts; the RI used to deal with partially vague or poorly characterized parameters [13].

RI has two features. First, the results are in the form of intervals. Second, the interval method doesn't ignore any part of solution region. Thus, the interval method gives a solution with high precision [3].

Youness [17] presented a nonlinear programming problem with a rough set of constraints. Also he defined the convex rough set, the local rough optimal solution, the global rough optimal solution, and the roughness measure of optimality.

$\mathrm{Xu}$ et al. [16] transformed from random rough nature to the equivalent crisp model and introduced an interactive method to get solution that satisfied decision maker, using a random rough simulation technique which can act with random rough objective functions and constraints, grouping with the genetic algorithm.

Osman et al. [9] introduced a new formulation and classification of the Rough Programming Problems. Also he discussed the rough feasibility, the rough optimality, the rough optimal value, and the rough optimal set.

Lu et al. [6] introduced the concept of RI to express dual uncertain information of many parameters and the related solution method presented to solve RI fuzzy linear programming problems.

Alolyan [1] tackled LP problems with fuzzy parameters in the objective function and the constraints based on preference relations between explored intervals.

Jana et al. [4] handled fuzzy rough multi-item economic production quantity model and developed constant demand. Infinite production rate has adaptability and dependability consideration in the production process, demand dependent unit production cost and shortages under the limitations on the capacity region, by geometric programming technique tackled the problem.

Hamazehee et al. [3] introduced a new class of LP problems in which some or all of the coefficients are RI and showed that each one of them can be transformed into two LP problems with interval coefficients. Also he introduced the surely optimal range, the possibly optimal range, the completely satisfactory solutions, the rather satisfactory solutions, and the rough optimal range.

Ammar and Khalifa [2] applied a new method named, separation method for solving Rough Interval Multi Objective Transportation Problems (RIMOTP), where transportation cost, supply, and demand are RIs. Also discussed that the separation method as an important tool for the DMs when they are handling various types of logistic problems having RI parameters of transportation problems.

Saad et al. [15] presented an algorithm for solving a three-level quadratic programming, where some or all of its coefficients in the objective function are RI.

Omran et al. [7] presented an algorithm for solving a three level fractional programming problem with rough coefficient in constraints.

Osman et al. [8] presented a solution approach for RIMOTP. The concept of solving conventional 
interval programming combined with fuzzy programming is used to build the solution approach for RIMOTP.

Pandian et al. [11] considered that transportation problem has all or some parameters as rough integer intervals. Also he proposed a new method named, a slice-sum method to solve Rough Integer Interval Transportation Problem, where transportation cost, supply, and demand are rough integer intervals.

\section{Formulation of Linear Programming Problem with Rough Interval Coefficients}

The linear programming problem with rough interval coefficients can be formulated as follows:

$\operatorname{Max} / \operatorname{Minf}(x)=\sum_{j=1}^{n}\left[\left[\underline{c}_{j}^{L}, \underline{c}_{j}^{U}\right],\left[\bar{c}_{j}^{L}, \bar{c}_{j}^{U}\right]\right] x_{j}$,

Subject to

$$
\begin{gathered}
G=\sum_{j=1}^{n}\left[\left[\underline{a}_{i j}^{L}, \underline{a}_{i j}^{U}\right],\left[\bar{a}_{i j}^{L}, \bar{a}_{i j}^{U}\right]\right] x_{j} \leq\left[\left[\underline{b}_{i}^{L}, \underline{b}_{i}^{U}\right],\left[\bar{b}_{i}^{L}, \bar{b}_{i}^{U}\right]\right], \\
\mathrm{i}=1,2, \ldots, \mathrm{m}, \mathrm{x}_{\mathrm{j}} \geq 0 .
\end{gathered}
$$

In the above Problem (1) - (2), $\left[\left[\underline{c}_{j}^{L}, \underline{c}_{j}^{U}\right],\left[\bar{c}_{j}^{L}, \bar{c}_{j}^{U}\right]\right]$ are RI coefficients of the objective function, $\left[\left[\underline{a}_{i j}^{L}, \underline{a}_{i j}^{U}\right],\left[\bar{a}_{i j}^{L}, \bar{a}_{i j}^{U}\right]\right]$ are RI coefficients of the constraints and $\left[\left[\underline{b}_{i}^{L}, \underline{b}_{i}^{U}\right],\left[\bar{b}_{i}^{L}, \bar{b}_{i}^{U}\right]\right]$ are RI of constants.

\section{Basic Preliminaries about RI}

Conversion of Linear Programming (LP) problem with rough interval coefficients into upper and lower approximation is usually hard work for many cases, but transformation process needs the following definitions to be known:

\section{Definition 1 [3]}

RI can be considered as a qualitative value from vague concept defined on a variable $\mathrm{x}$ in $\mathrm{R}$.

\section{Definition 2 [3]}

The qualitative value $A$ is called a rough interval when one can assign two closed intervals $A_{*}$ and $A^{*}$ on $R$ to it where $\mathrm{A}_{*} \subseteq \mathrm{A}^{*}$.

\section{Definition 3 [3]}

$A_{*}$ and $A^{*}$ are called the Lower Approximation Interval (LAI) and the Upper Approximation Interval (UAI) of $A$, respectively. Further, $A$ is denoted by $A=\left(A_{*}\right.$ and $\left.\mathrm{A}^{*}\right)$.

\section{Definition 4 [3]}

Consider all of the corresponding Linear Programming with Interval Coefficients (LPIC) and LP of Problem (1) - (2):

1) The interval $\left[f_{*}^{L}, f_{*}^{U}\right]\left(\left[f^{* L}, f^{* U}\right]\right)$ is called the surely (possibly) optimal range of Problem (1) (2) if the optimal range of each LPIC is a subset (superset) of $\left[f_{*}^{L}, f_{*}^{U}\right]\left(\left[f^{* L}, f^{* U}\right]\right)$.
2) Let $\left[f_{*}^{L}, f_{*}^{U}\right]\left(\left[f^{* L}, f^{* U}\right]\right)$ be surely (possibly) an optimal range of Problem (1) - (2), then the rough interval $\left[\left[f_{*}^{L}, f_{*}^{U}\right]\left[f^{* L}, f^{* U}\right]\right]$ is called the rough optimal range of Problem (1) - (2).

3) The optimal solution of each corresponding LPIC of Problem (1) - (2) whose optimal value belongs to $\left[f_{*}^{L}, f_{*}^{U}\right]\left(\left[f^{* L}, f^{* U}\right]\right)$ is called a completely (rather) satisfactory solution of Problem (1) - (2).

Let $\mathrm{D}$ denotes the set of all RIs on the real line $\mathrm{R}$. That is, $D=$

$\{[[b, c],[a, d]], a \leq b \leq c \leq$

$d$ and $a, b, c, d$ are in $R\}$,

$$
\begin{aligned}
& A=\left[\left[a_{2}, a_{3}\right],\left[a_{1}, a_{4}\right]\right] \text { and } B= \\
& {\left[\left[b_{2}, b_{3}\right],\left[b_{1}, b_{4}\right]\right] \text { be in } D .}
\end{aligned}
$$

\section{Definition 5 [11]}

Addition:

$A \oplus B=\left[\left[a_{2}+b_{2}, a_{3}+b_{3}\right],\left[a_{1}+b_{1}, a_{4}+\right.\right.$ $\left.\left.b_{4}\right]\right]$.

\section{Definition 6 [3]}

Subtraction:

$A-B=\left[\left[a_{2}-b_{3}, a_{3}-b_{2}\right],\left[a_{1}-b_{4}, a_{4}-\right.\right.$

$\left.\left.b_{1}\right]\right]$.

\section{Definition 7 [3]}

Negation: $-A=\left[\left[-a_{3},-a_{2}\right],\left[-a_{4},-a_{1}\right]\right]$,

Definition 8 [11]

Scalar Multiplication:

$K A=\left[\left[K a_{2}, K a_{3}\right],\left[K a_{1}, K a_{4}\right]\right]$,

if $\mathrm{k}$ is a positive real number.

\section{Definition 9 [11]}

Multiplication:

$A \otimes B=$

$\left[\left[a_{2} b_{2}, a_{3} b_{3}\right],\left[a_{1} b_{1}, a_{4} b_{4}\right]\right]$, if $A, B \geq 0$.

\section{Definition 10 [11]}

$A$ is said to be a rough positive integer if $a_{i}, i=$ $1,2,3,4$ are positive integers.

\section{Definition 11 [11]}

$A \geq B$, if $a_{i} \geq b_{i}, i=1,2,3,4$.

$A \leq B$, if $a_{i} \leq b_{i}, i=1,2,3,4$.

$A=B$, if $a_{i}=b_{i}, i=1,2,3,4$.

\section{Interval Method for Transforming RI Parameters to Crisp Nature}

Interval method [3] constructs two LP problems with interval coefficients as shown in Table (1). One of these problems is an LP where all of its coefficients are upper approximations of RI and the other is an LP where all of its coefficients are lower approximations of RI. 
Table (1): Two LP Problems with Lower and Upper Approximations of RI

\begin{tabular}{|c|c|}
\hline LP with Lower Approximations of RI & LP with Upper Approximations of RI \\
\hline $\begin{array}{l}\operatorname{Max} / \operatorname{Min} \underline{f}(x)=\sum_{j=1}\left[\underline{c}_{j}^{L}, \underline{c}_{j}^{U}\right] x_{j} \\
\text { Subject to } \\
\underline{G}=\sum_{j=1}^{n}\left[\underline{a}_{i j}^{L}, \underline{a}_{i j}^{U}\right] x_{j} \leq\left[\underline{b}_{i}^{L}, \underline{b}_{j}^{U}\right] \\
i=1,2, \ldots, m, x_{j} \geq 0\end{array}$ & $\begin{array}{l}\operatorname{Max} / \operatorname{Min} \bar{f}(x)=\sum_{j=1}\left[\bar{c}_{j}^{L}, \bar{c}_{j}^{U}\right] x_{j} \\
\quad \text { Subject to } \\
\bar{G} \quad=\sum_{j=1}^{n}\left[\bar{a}_{i j}^{L}, \bar{a}_{i j}^{U}\right] x_{j} \leq\left[\bar{b}_{i}^{L}, \bar{b}_{i}^{U}\right] \\
i=1,2, \ldots, m, x_{j} \geq 0\end{array}$ \\
\hline
\end{tabular}

Now, the equivalent problem of the LP problem with lower approximations of RI using interval method [3] can be obtained by getting the surely optimal range of
Problems (1) - (2), which resulted in the following two LP problems with crisp parameters in Table (2).

Table (2): Lower Approximations of RI

\begin{tabular}{|c|c|}
\hline Lower Approximation Lower Bound (LALB) & Lower Approximation Upper Bound (LAUB) \\
\hline $\begin{array}{l}\operatorname{Max} / \operatorname{Min} \underline{f}^{L}=\sum_{j=1} \underline{c}_{j}^{L} x_{j} \\
\text { Subject to } \\
\underline{G}^{L}=\sum_{j=1}^{n} \underline{a}_{i j}^{U} x_{j} \leq \underline{b}_{i}^{L}, i=1,2, \ldots, m, x_{j} \geq 0\end{array}$ & $\begin{array}{l}\operatorname{Max} / \operatorname{Min} \underline{f}^{U}=\sum_{j=1}^{n} \underline{c}_{j}^{U} x_{j} \\
\text { Subject to } \\
\underline{G}^{U}=\sum_{j=1}^{n} \underline{a}_{i j}^{L} x_{j} \leq \underline{b}_{i}^{U}, \quad i=1,2, \ldots, m, x_{j} \geq 0 .\end{array}$ \\
\hline
\end{tabular}

Now, the equivalent problem of the LP problem with upper approximations of RI using interval method [3] can be obtained by getting the possibly optimal range of Problems (1) - (2), which resulted in the following two LP problems with crisp parameters as shown in Table (3).

Table (3): Upper Approximations of RI

\begin{tabular}{|c|c|}
\hline Upper Approximation Lower Bound (UALB) & Upper Approximation Upper Bound (UAUB) \\
\hline $\operatorname{Max} / \operatorname{Min} \bar{f}^{L}=\sum_{j=1}^{n} \bar{c}_{j}^{L} x_{j}$ & $\operatorname{Max} / \operatorname{Min} \bar{f}^{U}=\sum_{j=1}^{n} \bar{c}_{j}^{U} x_{j}$ \\
\hline $\begin{array}{l}\text { Subject to } \\
\bar{G}^{L}=\sum_{j=1}^{n} \bar{a}_{i j}^{U} x_{j} \leq \bar{b}_{i}^{L}, i=1,2, \ldots, m, x_{j} \geq 0 .\end{array}$ & $\begin{array}{l}\text { Subject to } \\
\bar{G}^{U}=\sum_{j=1}^{n} \bar{a}_{i j}^{L} x_{j} \leq \bar{b}_{i}^{U}, i=1,2, \ldots, m, x_{j} \geq 0 .\end{array}$ \\
\hline
\end{tabular}

So, the LP problem with RI coefficients in the objective function and the constraints can be converted into four LP problems with crisp parameters.

\section{A Trust Probability Constraints for Transforming RI Parameters to Crisp Nature}

To convert the LP problem with rough coefficients in the objective functions into the respective crisp equivalents for solving a trust probability constraints, this process is usually hard work for many cases but the transformation process is introduced in the following theorem.

\section{Theorem 1[16]}

Assume that random rough variable $\tilde{c}_{i j}$ is characterized by $\tilde{c}_{\mathrm{ij}}(\lambda) \sim \mathrm{N}\left(\mathrm{c}_{\mathrm{ij}}(\lambda), \mathrm{V}_{\mathrm{i}}^{\mathrm{c}}\right)$ where:

$c_{i j}(\lambda)\left[\left(c_{i j}(\lambda)_{n \times 1}=\left(c_{i 1}(\lambda), c_{i 2}(\lambda), \ldots, c_{i n}(\lambda)\right)^{T}\right)\right]$ is a rough variable and $V_{i}^{c}$ is a positive definite covariance matrix. It follows thatc $c_{i}(\lambda)^{T} x=$ $([a, b],[c, d]), \quad($ wherec $\leq a \leq b \leq d) \quad$ is a rough variable and characterized by the following trust measure function: 


$$
\operatorname{Tr}\left\{c_{i}(\lambda)^{T} x \geq t\right\}= \begin{cases}0 & \text { if } d \leq t, \\ \frac{d-t}{2(d-c)} & \text { if } b \leq t \leq d, \\ \frac{1}{2}\left(\frac{d-t}{d-c}+\frac{b-t}{b-a}\right) & \text { if } a \leq t \leq b, \\ \frac{1}{2}\left(\frac{d-t}{d-c}+1\right) & \text { if } c \leq t \leq a, \\ 1 & \text { if } t \leq c .\end{cases}
$$

Then, we have $\operatorname{Tr}\left\{\lambda \mid \operatorname{pr}\left\{\mathrm{c}_{\mathrm{i}}(\lambda)^{\mathrm{T}} \mathrm{x} \geq \mathrm{F}_{\mathrm{i}}(\mathrm{x})\right\} \geq \delta_{\mathrm{i}}\right\} \geq \gamma_{\mathrm{i}}$, if and only if

$$
\begin{cases}b+R \leq F_{i} \leq d-2 \gamma_{i}(d-c)+R & \text { if } b \leq M \leq d, \\ a+R \leq F_{i} & \text { if } a \leq M \leq b, \\ \leq \frac{d(b-a)+b(d-c)-2 \gamma_{i}(d-c)(b-a)}{d-c+b-a} & \text { if } c \leq M \leq a, \\ +R & \text { if } M \leq c . \\ c+R \leq F_{i} \leq d-(d-c)\left(2 \gamma_{i}-1\right)+R & \end{cases}
$$

Where $\mathrm{M}=\mathrm{F}_{\mathrm{i}}-\phi^{-1}\left(1-\delta_{\mathrm{i}}\right) \sqrt{\mathrm{x}^{\mathrm{T}} \mathrm{V}_{\mathrm{i}}^{\mathrm{C}} \mathrm{x}}$ and $\mathrm{R}=$ $\phi^{-1}\left(1-\delta_{\mathrm{i}}\right) \sqrt{\mathrm{x}^{\mathrm{T}} \mathrm{V}_{\mathrm{i}}^{\mathrm{C}} \mathrm{x}}$, where $\phi \mathrm{is}$ the standardized normal distribution and $\delta_{\mathrm{i}}, \gamma_{\mathrm{i}} \in[0,1]$ are predetermined confidence levels.

\section{Fully Rough Intervals Problems}

Fully rough intervals problems [11], in which all decision parameters and decision variables in the objective functions and the constraints are RI and the optimal values of decision rough variables and rough objective functions are RI.

Formulation of Linear Programming Problem with Fully Rough Intervals

The linear programming problem with fully rough intervals coefficients can be formulated as follows: $\operatorname{Max} / \operatorname{Min}\left[\left[f^{L L}, f^{L U}\right],\left[f^{U L}, f^{U U}\right]\right]$

$$
\begin{aligned}
& =\sum_{j=1}^{n}\left[\left[\underline{c}_{j}^{L}, \underline{c}_{j}^{U}\right],\left[\bar{c}_{j}^{L}, \bar{c}_{j}^{U}\right]\right] \\
& \otimes\left[\left[x_{j}^{L L}, x_{j}^{L U}\right],\left[x_{j}^{U L}, x_{j}^{U U}\right]\right],
\end{aligned}
$$

Subject to

$$
\begin{aligned}
& G= \\
& \sum_{j=1}^{n}\left[\left[\underline{a}_{i j}^{L}, \underline{a}_{i j}^{U}\right],\left[\bar{a}_{i j}^{L}, \bar{a}_{i j}^{U}\right]\right] \otimes\left[\left[x_{j}^{L L}, x_{j}^{L U}\right],\left[x_{j}^{U L}, x_{j}^{U U}\right]\right] \leq \\
& {\left[\left[\underline{b}_{i}^{L}, \underline{b}_{i}^{U}\right],\left[\bar{b}_{i}^{L}, \bar{b}_{i}^{U}\right]\right], i=1,2, \ldots, m, \quad\left[X_{j}\right] \geq 0 .}
\end{aligned}
$$

In the above Problem (11) - (12) $\left[\left[x_{j}^{L L}, x_{j}^{L U}\right],\left[x_{j}^{U L}, x_{j}^{U U}\right]\right] \quad$ are $\quad \mathrm{RI}$ of decision variables, $\left[\left[\underline{c}_{j}^{L}, \underline{c}_{j}^{U}\right],\left[\bar{c}_{j}^{L}, \bar{c}_{j}^{U}\right]\right]$ are RI coefficients of the objective function, $\left[\left[\underline{a}_{i j}^{L}, \underline{a}_{i j}^{U}\right],\left[\bar{a}_{i j}^{L}, \bar{a}_{i j}^{U}\right]\right]$ are RI coefficients of the constraints and $\left[\left[\underline{b}_{i}^{L}, \underline{b}_{i}^{U}\right],\left[\bar{b}_{i}^{L}, \bar{b}_{i}^{U}\right]\right]$ are RI of constants.
Slice Sum Method for Transforming Fully Rough Intervals Linear Programming to Crisp Nature

Slice Sum method [11] is a method, in which the problem is sliced into four problems namely, UAUB problem, LAUB problem, LALB problem and UALB problem.

The transformation process is introduced in the following theorem.

\section{Theorem 2 [11]}

If $x_{j}^{* U U}, j=1,2, \ldots n$ is an optimal solution for the UAUB problem of the problem (11) - (12), $x_{j}^{* L U}, j=$ $1,2, \ldots n$ is an optimal solution for the LAUB problem of the problem $(11)-(12), x_{j}^{* L L}, j=1,2, \ldots n$ is an optimal solution for the LALB problem of the problem (11) - (12), $x_{j}^{* U L}, j=1,2, \ldots n$ is an optimal solution for the UALB problem of the problem (11) (12), then the set of RI $\left[\left[x_{j}^{* L L}, x_{j}^{* L U}\right],\left[x_{j}^{* U L}, x_{j}^{* U U}\right]\right]$ is an optimal solution for the problem (11) - (12) such that $x_{j}^{* U L} \leq x_{j}^{* L L} \leq x_{j}^{* L U} \leq x_{j}^{* U U}, j=1,2, \ldots n$.

To prove Theorem 2 above refer to [11].

The solution algorithm can be summarized in the following steps:

Step 1: Construct the UAUB problem of the given problem.

Step 2: Solve the UAUB problem using LP techniques. Let $x_{j}^{* U U}, j=1,2, \ldots n$ be an optimal solution of the UAUB problem.

Step 3: Construct the LAUB problem of the given problem.

Step 4: Solve the LAUB problem with upper bound constraints $\quad x_{j}^{L U} \leq x_{j}^{* U U}, j=1,2, \ldots n$ using $\quad$ LP techniques. Let $x_{j}^{* L U}, j=1,2, \ldots n$ be an optimal solution of the LAUB problem. 
Step 5: Construct the LALB problem of the given problem.

Step 6: Solve the LALB problem with upper bound constraints $\quad x_{j}^{L L} \leq x_{j}^{* L U}, j=1,2, \ldots$ nusing $\quad \mathrm{LP}$ techniques. Let $x_{j}^{* L L}, j=1,2, \ldots n$ be an optimal solution of the LALB problem.

Step 7: Construct the UALB problem of the given problem.

Step 8: Solve the UALB problem with upper bound constraints $\quad x_{j}^{U L} \leq x_{j}^{* L L}, j=1,2, \ldots$ nusing $\quad$ LP techniques. Let $x_{j}^{* U L}, j=1,2, \ldots n$ be an optimal solution of the UALB problem.

Step 9: The optimal solution of the given problem is $\left[\left[x_{j}^{* L L}, x_{j}^{* L U}\right],\left[x_{j}^{* U L}, x_{j}^{* U U}\right]\right], j=1,2, \ldots n$.

\section{On the Solution of a Rough Interval Three-level Quadratic Programming Problem}

A three-level quadratic programming problem is considered where some or all of its coefficients in the objective function are RI [15]. At the first phase of the solution approaches and to avoid the complexity of the problem, two QP problems with interval coefficients have been formulated. One of these problems was a QP where all of its coefficients are upper approximations of RI and the other problem was a QP where all of its coefficients are lower approximations of RI. At the second phase, a membership function is constructed to develop a fuzzy model for obtaining the optimal solution of the three-level quadratic programming problem.

\section{Problem Formulation and Solution Concept}

The Three-Level Quadratic Programming Problem with Rough Interval Coefficients (TLQPRIC) in the objective functions may be written as follows:

$$
\begin{aligned}
& {\left[1^{\text {st }} \text { Level }\right]} \\
& \operatorname{Max}_{\mathrm{x}_{1}} \mathrm{~F}_{1}(\mathrm{x}) \\
& =\sum_{\mathrm{j}=1}^{\mathrm{n}}\left[\left[\underline{\mathrm{a}}_{\mathrm{j}}^{\mathrm{L}}, \underline{\mathrm{a}}_{\mathrm{j}}^{\mathrm{U}}\right],\left[\overline{\mathrm{a}}_{\mathrm{j}}^{\mathrm{L}}, \overline{\mathrm{a}}_{\mathrm{j}}^{\mathrm{U}}\right]\right] \mathrm{x}_{\mathrm{j}} \\
& +\frac{1}{2} \mathrm{x}_{\mathrm{j}}^{\mathrm{T}}\left[\left[\underline{\mathrm{a}}_{\mathrm{j}}^{\mathrm{L}}, \underline{\mathrm{a}}_{\mathrm{j}}^{\mathrm{U}}\right],\left[\overline{\mathrm{a}}_{\mathrm{j}}^{\mathrm{L}}, \overline{\mathrm{a}}_{\mathrm{j}}^{\mathrm{U}}\right]\right] \mathrm{x}_{\mathrm{j}}, \\
& \quad \text { where } \mathrm{x}_{2}, \ldots, \mathrm{x}_{\mathrm{n}} \text { solves } \\
& {\left[2^{\mathrm{nd}} \operatorname{Level}\right]} \\
& \operatorname{Max}_{x_{2}} F_{2}(x) \\
& =\sum_{j=1}^{n}\left[\left[\underline{b}_{j}^{L}, \underline{b}_{j}^{U}\right],\left[\bar{b}_{j}^{L}, \bar{b}_{j}^{U}\right]\right] x_{j} \\
& +\frac{1}{2} x_{j}^{T}\left[\left[\underline{b}_{j}^{L}, \underline{b}_{j}^{U}\right],\left[\bar{b}_{j}^{L}, \bar{b}_{j}^{U}\right]\right] x_{j}, \\
& \quad \text { where } \mathrm{x}_{3}, \ldots, \mathrm{x}_{\mathrm{n}} \text { solves } \\
& {\left[3^{\text {rd }} \text { Level }\right]}
\end{aligned}
$$

$$
\begin{aligned}
& \operatorname{Max}_{x_{3}} F_{3}(x) \\
& =\sum_{j=1}^{n}\left[\left[\underline{c}_{j}^{L}, \underline{c}_{j}^{U}\right],\left[\bar{c}_{j}^{L}, \bar{c}_{j}^{U}\right]\right] x_{j} \\
& +\frac{1}{2} x_{j}^{T}\left[\left[\underline{c}_{j}^{L}, \underline{c}_{j}^{U}\right],\left[\bar{c}_{j}^{L}, \bar{c}_{j}^{U}\right]\right] x_{j}, \\
& \text { where } \mathrm{x}_{4}, \ldots, \mathrm{x}_{\mathrm{n}} \text { solves }
\end{aligned}
$$

Subject to

$G=$

$\{x \mid A x \leq d, x \geq 0\}$.

Where $G$ is the three-level convex constraint set, $F_{1}, F_{2}$ and $F_{3}$ are the objective functions of the FLDM, SLDM, TLDM, respectively. Also $\left[\left[\underline{a}_{j}^{L}, \underline{a}_{j}^{U}\right],\left[\bar{a}_{j}^{L}, \bar{a}_{j}^{U}\right]\right],\left[\left[\underline{b}_{j}^{L}, \underline{b}_{j}^{U}\right],\left[\bar{b}_{j}^{L}, \bar{b}_{j}^{U}\right]\right]$ and $\left[\left[\underline{c}_{j}^{L}, \underline{c}_{j}^{U}\right],\left[\bar{c}_{j}^{L}, \bar{c}_{j}^{U}\right]\right]$ are RI coefficients of the objective functions. Let $\quad(j=1,2, \ldots, n) \quad, x=$ $\left(x_{1}, x_{2}, \ldots, x_{n}\right)^{T}$ denote the vector of all decision variables.

A solution algorithm to solve the TLQPRIC problems (13) - (16) is described in a series of steps as follows [15]:

Step1: Determine the surly random rough interval coefficients range (lower (L) interval problem) in FLDM, SLDM, and TLDM problem, respectively.

Step2: Determine the possible random rough interval coefficients range (upper (U) interval problem) in FLDM, SLDM, and TLDM problem, go to Step3.

Step3: Formulate the corresponding equivalent threelevel quadratic programming problem.

Step4: Convert the lower and upper random interval coefficients in the FLDM problem into equivalent crisp models can be solved by classical methods.

Step5: Convert the lower and upper random interval coefficients SLDM, and TLDM problem into equivalent crisp models, go to Step 6.

Step6: Using the fuzzy approach as described in [10] to solve the resulting multi-level decision-making problems in Step 5.

Step7: Build membership functions of the FLDM, SLDM, and TLDM after determining the best and the worst solution of all lower interval coefficients and upper interval coefficients problems.

Step8: Solve a Tchebycheff problem [10] for all DMs level problem.

Step9: Control assumed the FLDM his /her decision by tolerance $t_{1}$.

Step10: Control assumed the SLDM his/her decision by tolerance $t_{2}$.

Step11: If $\omega<0$, increase $t_{1}, t_{2}$ then go to Step7; otherwise, go to Step 12.

Step12: The FLDM, SLDM, and TLDM calculating membership function $\grave{\mu}$.

Step13: Compute tolerance functions for $x_{1}, x_{2}$ using $t_{1}, t_{2}$ by [10]. 
Step14: Solve the Tchebycheff problem defined by [10], then go to Step15.

Step15: If the FLDM isn't satisfied with the solution then go to Step 9 with modifying, $\omega\left(\underline{\omega}^{L}, \underline{\omega}^{U}, \bar{\omega}^{L}, \bar{\omega}^{U}\right)$.

Step16: Stop.

\section{On Solving Three Level Fractional Programming Problem with Rough Coefficient in Constraints}

A three level fractional programming problem with a random rough coefficient in constraints was considered [7]. At the first phase of the solution approaches and to avoid the complexity of the problem, fractional programming problems were converted into a linear model problem using Charnes \& Cooper method. Then interval technique is used to convert the rough nature in constraints into the equivalent crisp model. At the final phase, a membership function was constructed to develop a fuzzy model for obtaining a compromised solution of the three level programming problems.

\section{Problem Formulation and Solution Concept}

The Three-Level Fractional Programming Problem with Rough Interval Coefficients (TLFPRIC) in the constraints may be written as follows:

[1st level]

$\operatorname{Max}_{x_{1}} F_{1}(x)=\frac{a_{1} x+\alpha_{1}}{b_{1} x+\beta_{1}}$,

where $\mathrm{x}_{2}$ solves

\section{[2nd level]}

$\operatorname{Max}_{\mathrm{x}_{2}} \mathrm{~F}_{2}(\mathrm{x})=\frac{\mathrm{a}_{2} \mathrm{x}+\alpha_{2}}{\mathrm{~b}_{2} \mathrm{x}+\beta_{2}}$,

where $x_{3}$ solves

[3rd level]

$\operatorname{Max}_{\mathrm{x}_{3}} \mathrm{~F}_{3}(\mathrm{x})=\frac{\mathrm{a}_{3} \mathrm{x}+\alpha_{3}}{\mathrm{~b}_{3} \mathrm{x}+\beta_{3}}$,

Subject to

$$
\begin{aligned}
& G=\left\{\sum_{j=1}^{n}\left(\left[a_{j}, b_{j}\right],\left[c_{j}, d_{j}\right]\right) x_{j} \leq l, x_{j} \geq 0, j\right. \\
& =1, \ldots, n\} \text {. }
\end{aligned}
$$

Where $F_{1}, F_{1}$ and $F_{3}$ are the objective functions of the FLDM, SDLM, and TLDM, $\left[\left[a_{j}, b_{j}\right],\left[c_{j}, d_{j}\right]\right]$ are RI coefficients of the constraints for the three levels.

A solution algorithm to solve the TLFPRIC problems (17) -(20) is described in a series of steps as follows [7]:

Step1: Fractional programming problems in the FLDM, SLDM, and TLDM were converted into a linear model problem using Charnes \& Cooper method. Step2: Determine the surly random rough interval coefficients range (lower (L) interval problem) and the possible random rough interval coefficients range (upper (U) interval problem) in FLDM, SLDM, and TLDM problem, respectively.

Step3: Formulate the corresponding equivalent three level fractional programming problems.

Step4: Convert the lower and upper random interval coefficients in the FLDM problem into equivalent crisp models can be solved by classical methods.

Step5: Convert the lower and upper random interval coefficients in the SLDM and TLDM problem into equivalent crisp models, go to Step 6.

Step6: Using the fuzzy approach as described in [10] to solve the resulting multi-level decision-making problems in Step 5.

Step7: Build membership functions of the FLDM, SLDM, and TLDM after determining the best and the worst solution of all lower interval coefficients and upper interval coefficients problems.

Step8: Solve a Tchebycheff problem [10] for all DMs level problem.

Step9: Control assumed the FLDM his /her decision by tolerance $t_{1}$.

Step10: Control assumed the SLDM his/her decision by tolerance $t_{2}$.

Step11: If $\omega<0$, increase $t_{1}, t_{2}$ then go to Step7; otherwise, go to Step 12.

Step12: The FLDM, SLDM, and TLDM calculating membership function $\grave{\mu}$.

Step13: Compute tolerance functions for $x_{1}, x_{2}$ using $t_{1}, t_{2}$ by [10].

Step14: Solve the Tchebycheff problem defined by [10], then go to Step15.

Step15: If the FLDM isn't satisfied with the solution then go to Step 9 with modifying, $\omega\left(\underline{\omega}^{L}, \underline{\omega}^{U}, \bar{\omega}^{L}, \bar{\omega}^{U}\right)$.

Step16: Stop.

\section{Fully Rough Integer Interval Transportation Problems}

A new method name, a Slice-Sum method for solving fully rough integer interval transportation problems was proposed [11]. The optimal values of decision rough variables and rough objective function for the problem that is obtained by the proposed method, were rough integer intervals.

\section{Problem Formulation and Solution Concept}

Consider the following fully rough integer transportation problem:

$$
\begin{aligned}
& \operatorname{Max} / \operatorname{Min}\left[\left[f^{L L}, f^{L U}\right],\left[f^{U L}, f^{U U}\right]\right] \\
& =\sum_{i=1}^{m} \sum_{j=1}^{n}\left[\left[\underline{c}_{i j}^{L}, \underline{c}_{i j}^{U}\right],\left[\bar{c}_{i j}^{L}, \bar{c}_{i j}^{U}\right]\right] \\
& \otimes\left[\left[x_{i j}^{L L}, x_{i j}^{L U}\right],\left[x_{i j}^{U L}, x_{i j}^{U U}\right]\right],
\end{aligned}
$$

Subject to

$$
\begin{aligned}
& \sum_{j=1}^{n}\left[\left[x_{i j}^{L L}, x_{i j}^{L U}\right],\left[x_{i j}^{U L}, x_{i j}^{U U}\right]\right]=\left[\left[\underline{a}_{i}^{L}, \underline{a}_{i}^{U}\right],\left[\bar{a}_{i}^{L}, a_{i}^{U}\right]\right], \\
& \mathrm{i}=1,2, \ldots, \mathrm{m},
\end{aligned}
$$


$\sum_{i=1}^{m}\left[\left[x_{i j}^{L L}, x_{i j}^{L U}\right],\left[x_{i j}^{U L}, x_{i j}^{U U}\right]\right]=\left[\left[\underline{b}_{j}^{L}, \underline{b}_{j}^{U}\right],\left[\bar{b}_{j}^{L}, \bar{b}_{j}^{U}\right]\right]$,

$\mathrm{j}=1,2, \ldots, \mathrm{n}$,

$\mathrm{x}_{\mathrm{ij}}^{\mathrm{LL}}, \mathrm{x}_{\mathrm{ij}}^{\mathrm{LU}}, \mathrm{x}_{\mathrm{ij}}^{\mathrm{UL}}, \mathrm{x}_{\mathrm{ij}}^{\mathrm{UU}} \geq 0$ and are integers. $\mathrm{i}=$ $1,2, \ldots$, mand $j=1,2, \ldots, n$.

In the above Problem (21) - (23), $c_{i j}^{L}, c_{i j}^{U}, \overline{\mathrm{c}}_{\mathrm{ij}}^{\mathrm{L}}$ and $\overline{\mathrm{c}}_{\mathrm{ij}}^{\mathrm{U}}$ are positive integers, $\underline{a}_{i}^{L}, \underline{a}_{i}^{U}, \bar{a}_{i}^{L}$ and $\bar{a}_{i}^{U}$ are positive integers, $\underline{b}_{j}^{L}, \underline{b}_{j}^{U}$, $\overline{\mathrm{b}}_{\mathrm{j}}^{\mathrm{L}}$ and $\overline{\mathrm{b}}_{\mathrm{j}}^{\mathrm{U}}$ are positive integers. The above problem is said to be balanced if the total supply is equal to the total demand.

An algorithm for solving the Fully Rough Integer Interval Transportation Problem [11]

Step1: Check that the given problem (21) - (23) is balanced. If not, make it into balanced.

Step2: Construct the UAUB integer transportation problem of the problem (21) - (23) .

Step3: Solve the UAUB integer transportation problem using a transportation algorithm [5] / the zeropoint method [12]. Let $x_{i j}^{* U U}, i=1,2, \ldots, m$ and $\mathrm{j}=1,2, \ldots, \mathrm{n}$ be an optimal solution of the UAUB integer transportation problem with the minimum transportation cost $f^{* U U}$.

Step4: Construct the LAUB integer transportation problem of the problem $(21)-(23)$.

Step5: Solve the LAUB integer transportation problem with upper bound constraints $x_{i j}^{L U} \leq$ $x_{i j}^{* U U}, i=1,2, \ldots, m$ and $j=1,2, \ldots, n$ using the zero point method [5] / the integer linear programing technique [12]. Let $x_{i j}^{* L U}, i=1,2, \ldots, m$ and $\mathrm{j}=$ $1,2, \ldots, n$ be an optimal solution of the LAUB integer transportation problem with the minimum transportation cost $f^{* L U}$.

Step6: Construct the LALB integer transportation problem of the problem (21) - (23).

Step7: Solve the LALB integer transportation problem with upper bound constraints $x_{i j}^{L L} \leq$ $x_{i j}^{* L U}, i=1,2, \ldots, m$ and $\mathrm{j}=1,2, \ldots, \mathrm{n}$ using the zero point method [5] / the integer linear programing technique [12]. Let $x_{i j}^{* L L}, i=1,2, \ldots, m$ and $\mathrm{j}=$ $1,2, \ldots, \mathrm{n}$ be an optimal solution of the LALB integer transportation problem with the minimum transportation cost $\mathrm{f}^{* \mathrm{LL}}$.

Step8: Construct the UALB integer transportation problem of the problem (21) - (23).

Step9: Solve the UALB integer transportation problem with upper bound constraints $x_{i j}^{U L} \leq$ $x_{i j}^{* L L}, i=1,2, \ldots, m$ and $\mathrm{j}=1,2, \ldots, \mathrm{n}$ using the zero point method [5] / the integer linear programing technique [12]. Let $x_{i j}^{* U L}, i=1,2, \ldots, m$ and $\mathrm{j}=$ $1,2, \ldots, \mathrm{n}$ be an optimal solution of the UALB integer transportation problem with the minimum transportation cost $\mathrm{f}^{* \mathrm{UL}}$.
Step10: The optimal solution of the given problem is $\left[\left[x_{i j}^{* L L}, x_{i j}^{* L U}\right],\left[x_{i j}^{* U L}, x_{i j}^{* U U}\right]\right]$ with the minimum transportation cost $\left[\left[f^{* L L}, f^{* L U}\right],\left[f^{* U L}, f^{* U U}\right]\right]$.

The reader is referred to Theorem 2 .

\section{Conclusion}

This paper stated a literature review on rough intervals formulation and methodologies such as formulation of linear programming problems with rough intervals coefficients in the objective functions and constraints, basic preliminaries about rough intervals, interval method and trust probability constraints for transforming rough intervals to crisp nature and fully rough intervals problems. Finally, presented different mathematical programming models that contain rough intervals coefficients.

\section{References}

[1] I. Alolyan, "Algorithm for Interval Linear Programming Involving Interval Constraints", Proceedings of the World Congress on Engineering and Computer Science, San Francisco USA, 10 (2013), 23-25.

[2] E. E. Ammar and A. M. Khalifa, "On Solving of Rough Interval Multi-objective Transportation Problems", Journal of Advances in Physics, 7 (2014), 1233-1244.

[3] A. Hamazehee, M. A. Yaghoobi and M. Mashinchi, "Linear Programming with Rough Interval Coefficients", Journal of Intelligent and Fuzzy Systems, 26 (2014), 1179-1189.

[4] D. K. Jana, K. Maity and T. K. Roy, "Multi-Item Production Inventory Model with Fuzzy Rough Coefficients via Geometric Programming Approach", OPSEARCH, 50 (2013), 475-490.

[5] H. S. Kasana and K. D. Kumar, "Introductory Operations Research: Theory and Applications", Springer International Edition, New Delhi, 2005.

[6] H. Lu, G. Huang and L. He, "An Inexact Rough Interval Fuzzy Linear Programming Method for Generating Conjunctive Water - Allocation Strategies to Agricultural Irrigation Systems", Applied Mathematics Modeling, 35 (2011), 4330-4340.

[7] M. Omran, O. E. Emam and A. S. Mahmoud, "On Solving Three Level Fractional Programming Problem with Rough Coefficient in Constraints", British Journal of Mathematics \& Computer Science, 12 (2015), 1-13.

[8] M. S. Osman, M. M. El-Sherbiny, H. A. Khalifa and H. H. Farag, "A Fuzzy Technique for Solving Rough Interval Multi-objective Transportation Problem", International Journal of Computer Applications, 147 (10) (2016), 49-57.

[9] M. S. Osman, E. F. Lashein, E. A. Youness, and T. E. M. Attya, "Mathematical Programming in Rough Environment", A Journal of Mathematical Programming and Operations Research, 60 (5) (2011), 603-611. 
[10] M. Osman, M. Abo-Sinna, A. Amer and O. Emam, "A Multi-Level Non-Linear Multi-Objective DecisionMaking under Fuzziness", Applied Mathematics and Computation, 153 (2004), 239-252.

[11] P. Pandian, G. Natarajan and A. Akilbasha, "Fully Rough Integer Interval Transportation Problems", International Journal of Pharmacy \& Technology, 8 (2) (2016), 13866-13876.

[12] P. Pandian, G. Natarajan, "A New Method for Finding An Optimal Solution for Transportation Problems", International Journal of Math. Sci.\& Engg. Appls, 4 (2010), 59-65.

[13] Z. Pawlak, J. Grzymala-Busse, R. Slowinski and W. Ziarko, "Rough Sets", Communications of the ACM, 38 (1995), 88-95.

[14] M. Rebolledo, "Rough Intervals-Enhancing Intervals for Qualitative Modeling of Technical Systems", Artificial Intelligence, 170 (2006), 667-685.

[15] O. M. Saad, O. E. Emam and Marwa M. Sleem, "On The Solution of a Rough Interval Three-level Quadratic Programming Problem", British Journal of Mathematics \& Computer Science, 5 (2015).

[16] J. Xu and L. Yao, "A Class of Multi-Objective Linear Programming Models with Random Rough Coefficients", Mathematical and Computer Modeling, 49(2009), 189-206.

[17] E. Youness, "Characterizing Solutions of Rough Programming Problems", European Journal of Operational Research, 168 (2006), 1019-1029. 